The first case of a myxoid liposarcoma metastatic to the tongue of a 78-yearold male patient with primary scrotal liposarcoma for the past seven years is reported. The patient presented with a firm exophytic mass of the left dorsal surface of the tongue, sized 4 to $4.5 \mathrm{~cm}$, with oedema and bleeding. Myxoid liposarcoma is a malignancy with relatively good prognosis and low metastatic rate but high local recurrence. This is the second case report recorded of a metastatic liposarcoma to the oral cavity. Clinical and imaging considerations, as well pathological details concerning the myxoid liposarcoma, are described.

Key words: myxoid liposarcoma, tongue, metastasis.

Contemp Oncol (Pozn) 2020; 24 (3): 203-205 DOI: https://doi.org/10.5114/wo.2020.97483

\section{Metastatic myxoid liposarcoma to the tongue: a unique intraoral metastasis}

\author{
Dimitris Tatsis ${ }^{1}$, Dimitris Deligiannidis ${ }^{1}$, Styliani Papaemmanouil ${ }^{2}$, \\ Konstantinos Vahtsevanos ${ }^{1}$
}

${ }^{1}$ Department of Oral and Maxillofacial Surgery, Aristotle University of Thessaloniki, Greece

2Department of Pathology, General Hospital of Thessaloniki "G. Papanikolaou”, Thessaloniki, Greece

\section{Introduction}

Soft-tissue sarcomas (STS) are rare malignancies, comprising less than $1 \%$ of malignant tumours in adults [1]. Liposarcoma is a type of STS deriving from the adipose tissue, and accounts for $15 \%$ of all STSs. It is the second most common STS after malignant fibrous histiocytoma (recently renamed as undifferentiated pleomorphic sarcoma) [2]. Liposarcoma has distinct histopathological subtypes; well differentiated, myxoid, pleomorphic, and dedifferentiated, with the first being the most common [3, 4].

Liposarcoma is frequently located in the lower limbs and retroperitoneum, although it can rarely be found in the head and neck region. Less than $1 \%$ of primary liposarcomas are located in the head and neck, and less than $0.3 \%$ are located in the oral region $[3,5]$. A recent systematic review gathered 104 primary liposarcomas of the oral cavity [5-8]. Even fewer reports of metastatic liposarcomas to the head and neck region have been published. A recent literature review comprised 16 reports [9].

The aim of this paper is to present a case of metastatic myxoid liposarcoma to the tongue of a patient with primary scrotal location, recording the first patient with metastasis of liposarcoma to the tongue.

\section{Case report}

A 78-year-old patient presented with a firm exophytic mass of the left dorsal surface of the tongue, sized $4 \times 4.5 \mathrm{~cm}$ (Fig. 1). Mild oedema and light bleeding were his main symptoms. The patient had a history of myxoid liposarcoma of the scrotum with two surgical excisions seven and six years ago and concomitant radiotherapy and chemotherapy with ifosfamide-etoposide-mesna. Two years later the patient had a lung metastasis and received immunotherapy with trabectedin, but due to adverse reactions, he continued treatment with targeted therapy (pazopanib) up to the day of examination in our department.

An MRI was performed, describing an exophytic solid mass on the dorsal surface of the tongue sized $4-4.5 \mathrm{~cm}$ (Fig. 2). A biopsy was taken, and the histopathology report presented a malignant neoplastic process with morphological features of a myxoid liposarcoma, with uniformly round to oval shaped primitive non-lipogenic mesenchymal cells and signet-ring lipoblasts in a prominent myxoid stroma, with rich "chicken-wire" capillary vasculature (Figs. 3 and 4). The patient was referred to his oncologist for systemic evaluation and therapy.

\section{Discussion}

A typical presentation of a liposarcoma is a slow growing, firm, yellowish mass, well circumscribed, most commonly appearing in the fourth to 


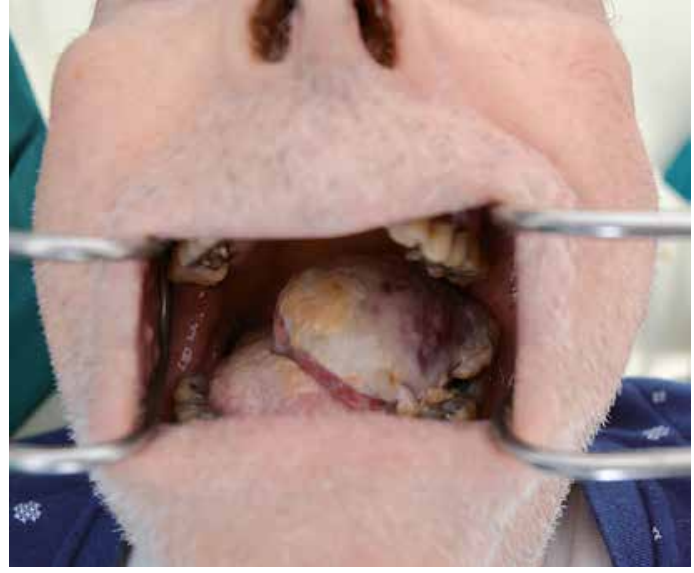

Fig. 1. Clinical presentation. Published with the patient's consent

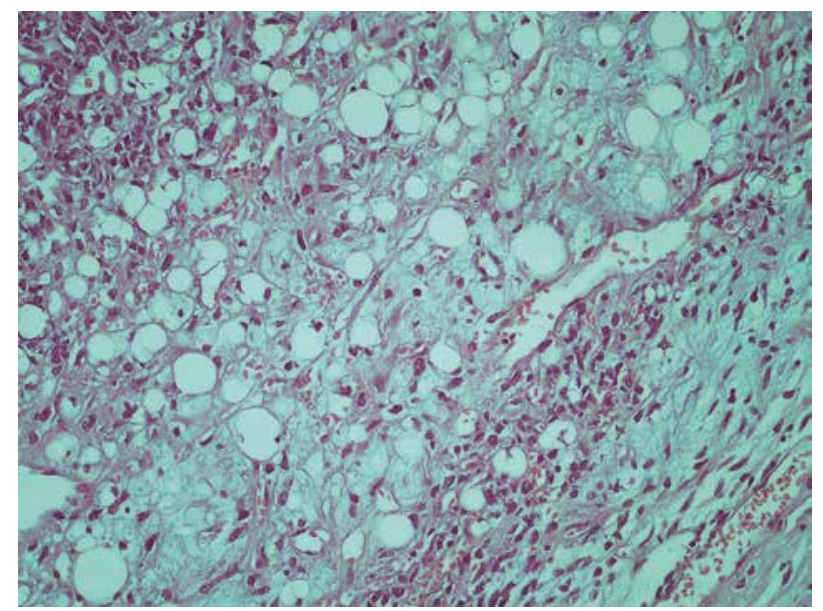

Fig. 3. A mixture of uniform round to oval-shaped non-lipogenic cells and small signet ring lipoblasts in a myxoid stroma (haematoxylin and eosin, $\times 200$ )

sixth decade of life. Signs of necrosis with haemorrhagic features are not uncommon. Myxoid liposarcoma is the commonest type and is believed to have the mildest biological behaviour because the rate of distant metastasis is considered low. Myxoid liposarcoma has a relatively good prognosis, with a five-year survival rate of $80-90 \%$. In liposarcomas of the oral cavity, survival rates are calculated at $66.4 \%$ [8]. There is a high incidence of local recurrence of up to $33 \%$ [2, 5]. In general, liposarcomas tend to metastasise to the lungs, whereas the myxoid type can appear with extrapulmonary metastasis [2, 10]. Nodal metastases are rare with primary liposarcomas of the head and neck region, as well as with any type of primary liposarcoma [7, 11]. A systematic review published on primary oral liposarcomas reported a $2 \%$ incidence of distal metastases [8].

The subcutaneous tissue of the head and neck is considered the most common site of head and neck liposarcomas. The most common sites of primary intraoral liposarcomas are the tongue, cheek, and buccal mucosa. Reports have been published on cases affecting the floor of the mouth, lip, palate, and gingiva [8, 12].

According to McElderry et al. [9], very few cases of metastatic liposarcomas to the head and neck region have been

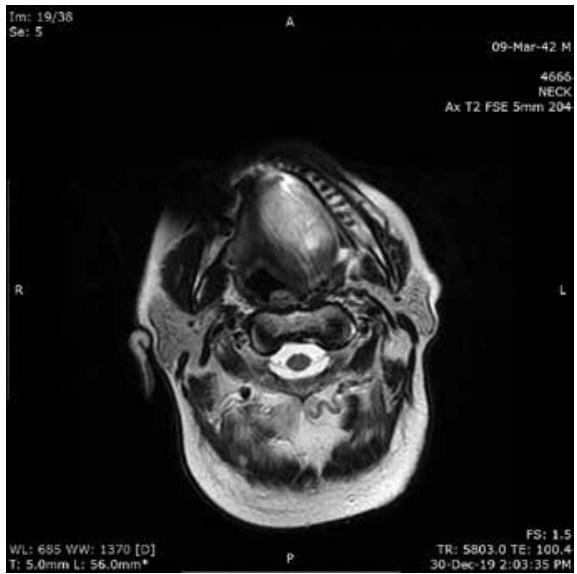

Fig. 2. MRI, T2-weighted image, axial plane. A lesion sized $4-4.5 \mathrm{~cm}$ on the dorsal surface of the tongue is imaged

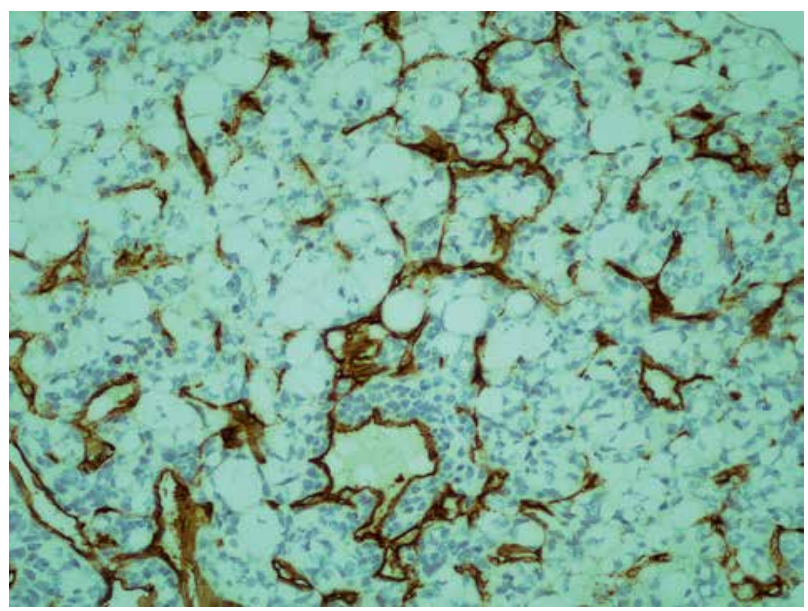

Fig. 4. A rich of delicate, arborising "chicken-wire" capillary vasculature (immunostain CD34, ×200)

published. Their case concerns a dedifferentiated liposarcoma of the left shoulder of a 68-year-old male patient metastasising to the gingival mucosa. This seems to be the only intraoral metastatic liposarcoma recorded. The remaining 15 cases recorded concern metastasis to the orbits (five cases), thyroid (four cases), dura mater (three cases), submandibular region (one case), neck (one case), and scalp (one case).

Pathological diagnosis of a liposarcoma in the head and neck region may be challenging for the histopathologist due to the rarity of this location. A wide differential diagnosis can be included; myxoma, myxosarcoma, myxofibrosarcoma, lipoma, or undifferentiated pleomorphic sarcoma [2, 8]. In our case, the pathological specimen had all the typical characteristics of a myxoid liposarcoma, providing a definitive diagnosis for the patient.

Typical therapy of a liposarcoma is a wide surgical excision. As far metastatic sites are concerned, data are very limited. From the aforementioned cases, nine were managed surgically, and one received radiotherapy only. The remaining reports do not define the course of therapy. Our patient could not be managed surgically due to the size of the lesion. Thus, the decision of referring the patient to his oncologist for continuation of systemic therapy was made. 


\section{Conclusions}

In conclusion, with this report we add a new metastatic location of a myxoid liposarcoma to the literature, recording a patient with metastatic myxoid liposarcoma to the tongue, with primary location to the scrotum. To our knowledge, this is the first reported case of a myxoid liposarcoma metastasis to the tongue, and the second report of any type of liposarcoma metastasis to the oral cavity.

The authors declare no conflict of interest.

\section{References}

1. Zahm SH, Fraumeni JF. The epidemiology of soft tissue sarcoma. Semin Oncol 1997; 24: 504-514.

2. Zagzoog N, Ra G, Koziarz A, Provias J, Sommer D, Almenawer SA, Reddy K. Metastatic liposarcoma of the skull base: A case report and review of literature. Neurosurgery 2017; 80: 219-223.

3. Angiero F, Sidoni A, Stefani M. Liposarcoma of the oral cavity case reports of the pleomorphic and the dedifferentiated variants and a review of the literature. Anticancer Res 2006; 26: 4857-4867.

4. Jo VY, Fletcher CDM. WHO classification of soft tissue tumours: an update based on the 2013 (4th) edition. Pathology 2014; 46: 95-104.

5. Fanburg-Smith JC, Furlong MA, Childers ELB. Liposarcoma of the oral and salivary gland region: A clinicopathologic study of 18 cases with emphasis on specific sites, morphologic subtypes, and clinical outcome. Mod Pathol 2002; 15: 1020-1031.

6. Minić AJ. Liposarcomas of the oral tissues: a clinicopathologic study of four tumors. J Oral Pathol Med 1995; 24: 180-184.

7. Zheng JW, Wang Y. Liposarcoma in the oral and maxillofacial region: An analysis of 10 consecutive patients. J Oral Maxillofac Surg 1994; 52: 595-598.

8. Pontes FSC, de Souza LL, Vulcão ÉNC, de Abreu MC, Menezes SN, Rezende D, Pontes HAR. Liposarcoma of oral cavity: Systematic review of cases reported to date and analysis of prognostic factors. Head Neck 2020 Apr 20. doi: 10.1002/hed.26160 [Online ahead of print].

9. McElderry J, McKenney JK, Stack BC. High-grade liposarcoma metastatic to the gingival mucosa: case report and literature review. Am J Otolaryngol 2008; 29: 130-134.

10. Tysome JR, Sandison A, Clarke PM. Myxoid liposarcoma metastatic to the thyroid gland: A case report and literature review. I Laryngol Otol 2006; 120: 511-513.

11. Fong Y, Coit DG, Woodruff JM, Brennan MF. Lymph node metastasis from soft tissue sarcoma in adults: Analysis of data from a prospective database of 1772 sarcoma patients. Ann Surg 1993; 217: 72-77.

12. Agarwal J, Kadakia S, Agaimy A, Ogadzanov A, Khorsandi A, Chai RL. Pleomorphic liposarcoma of the head and neck: Presentation of two cases and literature review. Am J Otolaryngol 2017; 38: 505-507.

\section{Address for correspondence}

\section{Dimitris Tatsis}

Department of Oral and Maxillofacial Surgery

Aristotle University of Thessaloniki

Exochi, 57010, Thessaloniki, Greece

e-mail: dtatsis@outlook.com

Submitted: 24.05 .2020

Accepted: 10.06 .2020 